\title{
Circular paraboloid reflection cell for laser spectroscopic trace gas analysis
}

\author{
Markus Mangold,${ }^{1,2, *}$ Béla Tuzson, ${ }^{1}$ Morten Hundt, ${ }^{1}$ Jana Jágerská,,${ }^{1,3}$ \\ HERBERT LOOSER, ${ }^{4}$ AND LUKAs EMMENEGger ${ }^{1}$ \\ ${ }^{1}$ Swiss Federal Laboratories for Materials Science and Technology, Empa, Überlandstrasse 129, 8600 Dübendorf, Switzerland \\ ${ }^{2} I R s w e e p \mathrm{GmbH}$, c/o ETH Zürich, IQE, Auguste-Piccard-Hof 1, HPT H4.1, 8093 Zürich, Switzerland \\ ${ }^{3}$ Department of Physics and Technology, UiT The Arctic University of Norway, 9037 Troms $\varnothing$, Norway \\ ${ }^{4}$ FHNW, University of Applied Sciences, Institute for Aerosol and Sensor Technology, Steinackerstrasse 5, Windisch 5210, Switzerland \\ *Corresponding author: markus.mangold@irsweep.com
}

\begin{abstract}
Absorption cells with circular geometry are a class of multipass reflection cells consisting of a single, circular mirror. They can be particularly favorable for trace gas measurements because of their mechanical robustness, simplicity, and their optical versatility. In this article, we present detailed theoretical considerations and ray tracing simulations for the optimization of the optical design of circular multipass reflection cells. A parabolic mirror shape in a confocal arrangement is found to be most suitable for long optical paths in a small volume. We experimentally demonstrate more than $12 \mathrm{~m}$ optical path in a $14.5 \mathrm{~cm}$ diameter gas cell and $\mathrm{NO}_{2}$ concentration measurements in ambient air with a measurement precision better than $0.1 \mathrm{ppb}$.
\end{abstract}

OCIS codes: (140.3490) Lasers, distributed-feedback; (300.6360) Spectroscopy, laser; (080.4035) Mirror system design

http://dx.doi.org/10.1364/JOSAA.33.000913

Keeping track of trace gas levels is of outstanding importance in a wide range of applications. In industry, trace gas monitoring is essential for process control as well as for work safety reasons [1]. In the medical domain, human breath analysis is rapidly evolving as a non-invasive method of disease recognition and diagnostics [2,3]. Last, but not least, most trace gases have a strong impact on our environment; reactive species are often toxic or change the atmospheric chemistry and composition, while many stable molecules influence the Earth's climate due to their radiative forcing. Hence, long-term trace gas monitoring is of highest priority. Infrared laser spectroscopy is a powerful tool to monitor trace gas species in a large variety of applications. Especially in the mid-infrared spectral range, where most molecules exhibit strong absorption features, trace gas sensors can achieve high sensitivity and selectivity [4].

For low abundance trace gases, the small absorption signals are usually compensated for by increasing the optical path length through the sample, either by the use of multipass cells or cavity-enhanced methods. In cavity-enhanced spectroscopy, extremely long optical paths on the order of several $\mathrm{km}$ can be achieved within high-finesse optical cavities [5]. However, cavity-enhanced techniques are demanding in many respects, such as the high reflectivity of the mirrors, the avoidance of feed-back to the laser or matching of the cavity and the laser frequency.

Multipass cells have a long history in laser spectroscopy. They usually allow for broad spectral bandwidth, and numerous designs have been proposed in the search for long path length, small footprint, simple alignment, robustness, and low production cost. The oldest, wellestablished cell design is the white cell relying on three identical spherical mirrors placed in a nearly confocal mirror arrangement [6]. It is still widely used due to its simple optical elements, but the threemirror arrangement results in a rather large volume. The Herriott cell consists of only two spherical mirrors in a nearly confocal configuration
[7]. Further variations such as the astigmatic Herriott cell $[8,9]$ and the split mirror cell [10] illustrate the great success of this geometry.

A new category of multipass cells was introduced by Thoma and coworkers, which is generally referred to as circular multi- pass reflection cells [11]. Such cells rely on a circular arrangement of spherical mirrors. In some studies, multiple spherical mirrors were positioned on a circle in a concentric arrangement [11-15]. Recently, several research groups have introduced multipass cells consisting of only a single, circular mirror [16-20]. In these cells, the cell body is, at the same time, the mirror reflecting the optical beam. The advantage of such a configuration lies in its robustness toward mechanical stress such as vibrations or temperature changes. Furthermore, com-pact setups are possible [21].

The present article is focused on the systematic design and optimization of absorption cells based on a circular geometry. First, we describe the various design parameters such as mirror arrangement, mirror shape, and optical beam pattern on a theoretical basis. Then, we compare different designs using ray tracing simulations. Finally, we present laser spectroscopic measurements using an optimized multipass cell design with a parabolic surface shape allowing for up to $12 \mathrm{~m}$ optical path. Performance of the optical setup is characterized for ambient air $\mathrm{NO}_{2}$ concentration measurements.

The basic principle of circular multipass cells relies on the fact that a beam entering a circular mirror under an angle propagates along a starshaped pattern. The actual pattern described by the beam is defined by the incidence angle $\Theta$ under which the beam enters the circular mirror. The pattern is also known as a star polygon, which can be described by two parameters: the number of spikes $p$ and the density $q$ of the star. The former defines how often the beam passes through the cell before reaching the entrance again. The latter indicates how close to the center the beams pass through the cell. 


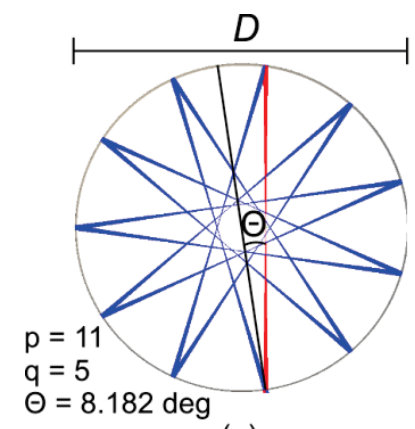

(a)

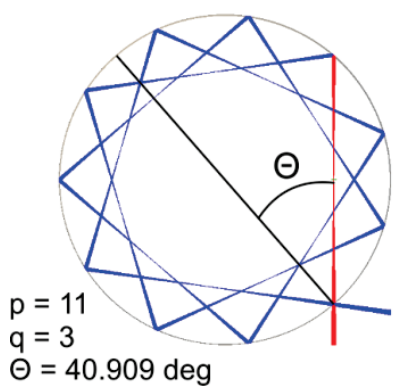

(c)

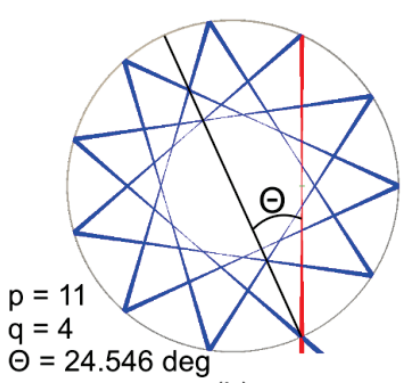

(b)

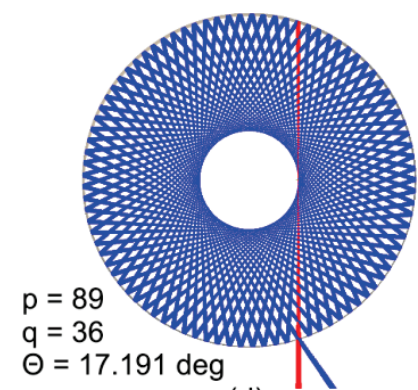

(d)
Figure 1: Star polygons. Inside a circular mirror, a beam propagates on a closed star-shaped beam pattern depending on the incidence angle $\Theta$.11-spiked stars can be produced under several different incidence angles by changing the star's density (a) $q=5$, (b) $q=4$, (c) $q=3$. (d) 89-spiked star pattern with density $q=36$ used in our experimental setup.

For an arbitrary star polygon, the incidence angle can be calculated as a function of $p$ and $q[22]$ :

$$
\Theta=90^{\circ} \cdot\left(1-\frac{2 q}{p}\right)
$$

Figures 1(a)-1(c) illustrate 11-spiked star patterns with various $q$ values. It can be seen that the same number of optical beam passes can be achieved under completely different $\Theta$ values of 8.182, 24.546, and 40.909 deg. Conversely, very different numbers of spikes $p$ (corresponding to large variations of the path length in a given circular cell) can be achieved under nearly identical angles by keeping the $q / p$ ratio constant.

Finally, the $q / p$ ratio decides on the beam pattern that is generated under off-axis irradiation (see below). For practical reasons, the incidence angle $\Theta$ is chosen such that the incoming and outgoing beams can be easily separated. Furthermore, the number of reflections is limited by the reflectivity of the mirror and the beam waist, i.e., the ability to (re)focus the beam. As an illustration of a dense pattern, Fig. 1(d) shows an 89-spiked star with density $q 36$ in a $144 \mathrm{~mm}$ diameter cylinder. This configuration will also be used in the experimental setup described below.

With a given beam pattern, the length of a single pass through a cylinder is $l_{0}=D \cos (\Theta)$ thus the length of the complete optical path is

$$
l_{\text {opt }}=p \cdot D \cdot \cos (\Theta),
$$

where $D$ is the diameter of the cylinder. Using this formula, the complete optical path of the above-mentioned cell configuration is $12.24 \mathrm{~m}$. However, the very same reflection cell can be used in a 42spiked star configuration with an optical path of $5.78 \mathrm{~m}$. This is achieved by reducing the incidence angle as compared with the 89spiked configuration by only $0.048 \mathrm{deg}$. Conversely, by increasing the angle by $0.809 \mathrm{deg}$, a five-spiked star with an optical path of $68.5 \mathrm{~cm}$ is obtained. Thus, a given cell geometry can be used for very different applications requiring various path lengths.

Next, we consider the shape of the cell inner surface. As illustrated in Fig. 2(a), the cell is a ring with inner diameter $D$ and an arbitrarily shaped inner surface representing the reflective area, i.e., the mirror section. Considering the ray propagation across the system, we distinguish between sagittal and tangential planes. The sagittal plane is indicated in Figs. 2(a) and 2(b) in gray. Perpendicular to it resides the tangential plane in which the light beam propagates, as indicated by the red line in Fig. 2(b). Having a rotational symmetry, the radius of curvature in the tangential direction $r_{t}$ is always given by the diameter of the cylinder, i.e., $r_{t}=D / 2$. Contrarily, the surface shape in the sagittal direction can be freely chosen in the design.

The simplest shape of a cylindrical multipass cell is a straight cylinder, where $r_{s}=\infty$, as schematically shown in Fig. 2(c). While being of striking simplicity, the straight cylinder has some clear disadvantages as compared to bent surface shapes. In particular, the straight cylinder is very sensitive to not perfectly parallel surfaces and therefore needs tremendously high manufacturing quality. Furthermore, a divergence of the incoming beam in the sagittal direction will persist throughout the propagation of the beam, which strongly limits the maximum path length that can be obtained in such a configuration.

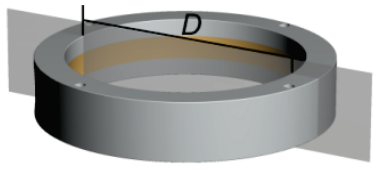

(a)

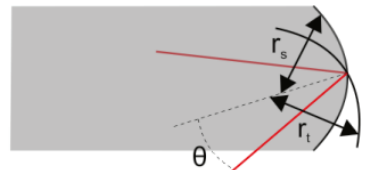

(b)

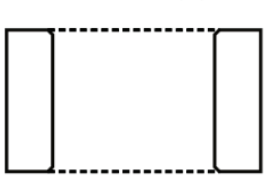

(c)

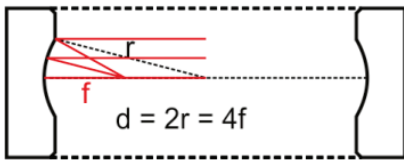

(f)

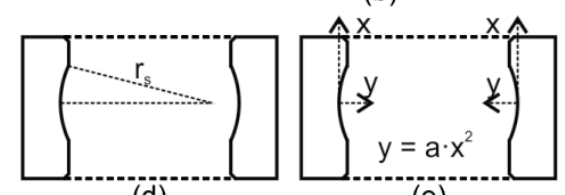

(d) (e)

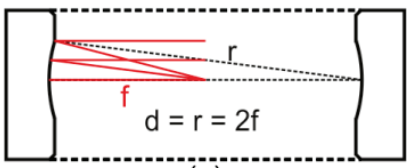

(g)
Figure 2: Mirror shapes and arrangements. (a) 3D view of a cylindrical multipass cell with inner diameter D. In gray, the sectioning plane in the sagittal direction used for subequent figures is indicated. (b) Schematic view of a toroidal mirror with tangential curvature rt and sagittal cur-vature rs, with a light beam in the tangential plane incident on the sur- face under an angel $\Theta$ indicated in red. Schematic sections of cylindrical cells illustrate the possible surface shapes (c) straight cylinder, (d) toroidal surface, and (e) parabolic surface. Depending on the focal length with respect to the distance a pair of mirrors can be placed in a (f) concentric or (g) confocal arrangement.

The propagation length can be increased considerably by adding a curvature to the mirror in the sagittal direction, as schematically shown in Fig. 2(d). In this situation, the two radii $r_{s}$ and $r_{t}$ describe a toroidal surface. As discussed in [19], when a light beam is reflected from a toroidal surface under an angle with respect to the surface normal, we have to account for astigmatism. For instance, a light beam travelling in the tangential plane incident on the surface under an angle $\Theta$ [see Fig. 2(b)] will have a different focal length in the sagittal and tangential direction $r_{s}=r_{t}$. To correct for that, the two radii have to fulfill Coddington's equations, i.e.

$$
\frac{r_{s}}{r_{t}}=\cos ^{2} \Theta
$$

In such a toroidal cylinder, the beam is refocalized to an aberration free focal point after every reflection, resulting in stable beam propagation over long distances. In reference [19], we have shown up to 51 reflections in a toroidal cell resulting in $408 \mathrm{~cm}$ optical path.

The surface shape in sagittal direction is, however, not limited to spherical geometry. In fact, todays fabrication methods allow us shaping the surface completely arbitrarily. Another useful surface shape is a parabolic geometry with $y=a x^{2}$ such as is schematically shown in Fig. 2(e). As long as the beam remains close to the center of the parabola (at $x=0$ ), a parabolic surface behaves just like a spherical surface, since they only differ in the third order of $x$. The actual appeal of a parabolic surface 
shape lies in its ideal imaging properties under non-centric illumination, as we will see later on. For the time being, we can just replace the curvature $r_{s}$ by the quadratic coefficient $a$, such that the focal length $f$ remains the same, i.e. $\frac{r_{s}}{2}=f=\frac{1}{4 a}$. To take into account spherical aberration, Coddington's equation for the parabolic case is then

$$
\frac{1}{2 r_{t} a}=\cos ^{2} \Theta
$$

Now, let us further consider two special types of mirror arrangements found in mirror cavities. The first one is the so called concentric arrangement, where the distance between the two mirrors is equal to twice their radius of curvature (see Fig. 2(f)). In such a cavity, a parallel beam would be focalized to $f=D / 4$. Contrarily, a point source at the center of the cavity (or equivalently a beam focalized to the center of the cavity) is refocalized exactly to the center of the cavity. It is known from laser cavities, that such a mirror arrangement is meta-stable, i.e. it is exactly at the border between stable and unstable cavities [cite laser spectroscopy book]. Obviously, a cylindrical cell is always in a concentric arrangement in tangential direction, since it needs to close to a circle. By forcing the focal length in sagittal direction to be equal to the focal length in tangential direction using equations (3) and (4), sagittal as well as tangential mirror direction are in a concentric mirror arrangement.

Alternatively, two spherical mirrors can be positioned such that their distance is equal to twice their focal length, resulting in a confocal arrangement (see Fig. 2(g)). Consequently, a parallel beam is focalized to the center of the cavity and is collimated again after anew reflection on a mirror. Also this configuration is meta-stable, however, only with respect to asymmetry and not with respect to changes in the focal length of the mirrors [cite laser spectroscopy book again]. As stated before, a cylindrical cell is always in concentric configuration in tangential direction. However, in sagittal direction we are free to choose the focal length and can easily reach a confocal arrangement by simply multiplying the left hand side of equations (3) and (4) with $\frac{1}{2}$. As we will see, such an arrangement leads to much more stable beam propagation in a cylindrical cell.

To evaluate different mirror shapes and arrangements we have performed raytracing simulations using FRED® optical engineering software (Photonics Engineering Inc.). The simulated optical setup is shown in Fig. 3(a). A Gaussian laser beam with $1 \mathrm{~mm} \mathrm{1/e-half} \mathrm{width} \mathrm{is}$ focused with a $150 \mathrm{~mm}$ reflected focal length off-axis parabolic mirror to the center of a cylindrical mirror. The mirror surface is assumed to have $98 \%$ reflectivity, corresponding to the reflectivity of gold in the mid-infrared spectral range [24]. Inside the cylindrical mirror we have placed a 1-mm thick cylinder with $4 \mathrm{~mm}$ diameter holes representing the absorption mask used for stray light suppression [25]. The surface of the mask is assumed to be a perfect absorber for mid-infrared light. The out-coupled beam is absorbed by a detector surface at a distance of $20 \mathrm{~mm}$ from the cylinder's entrance/exit aperture. The outcome of the simulations is a transmission of the optical configuration, defined as the ratio of the intensity arriving at this detector surface to the intensity initially emitted by the laser.

In Figure 3(b), we plot the transmission of the cylinder as a function of the focal length in sagittal direction. The blue line stems from simulations of a $140 \mathrm{~mm}$ diameter cylinder with a parabolic surface shape, while the orange line is the results obtained with a $140 \mathrm{~mm}$ diameter toroid. We find the same behavior for both surface shapes: the transmission is constant at $16.5 \%$ for weak curvature in sagittal direction (corresponding to long focal length). At a focal length of $31.938 \mathrm{~mm}$ we observe a very steep decrease of the transmission. This represents the meta-stability of a concentric mirror arrangement. For $r_{t}=70 \mathrm{~mm}$ we calculate $r_{s}=63.885 \mathrm{~mm}$ and thus $f_{s}=31.943 \mathrm{~mm}$ according to equation (3). We infer from this simulation that for a concentric toroidal mirror the tolerance in $r_{s}$ is $<10 \mu \mathrm{m}$. Conversely, for a confocal mirror arrangement, the accuracy of the sagittal curvature is of minor importance, since the transmission is basically not influenced by changing the curvature.
The higher stability of the confocal mirror arrangement also expresses via increased tolerance with respect to misalignment of the optical setup. In Fig. 3(c) we plot the transmission of a cylindrical mirror cell under rotation of the off-axis parabolic mirror used to focalize the input beam about the y-axis, which leads to a shift of the beam in sagittal direction. Figure 3(d) shows a zoom in on the same data. The blue line is obtained with a confocal, the orange line with a concentric mirror arrangement. We find that the confocal arrangement is 100 -fold more tolerant to rotation of the coupling mirror about the $y$-axis.

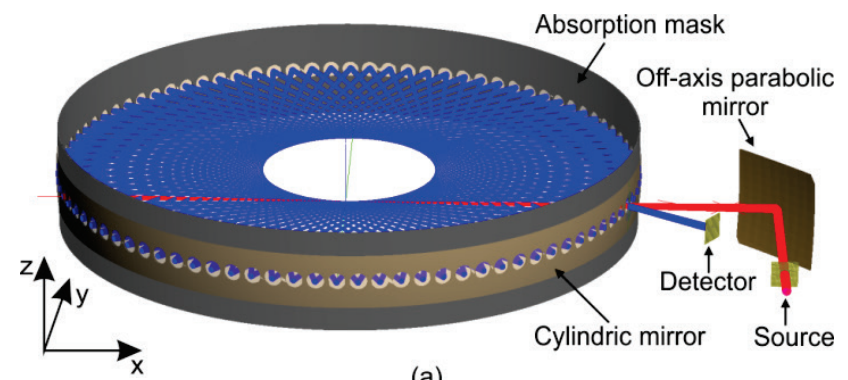

(a)

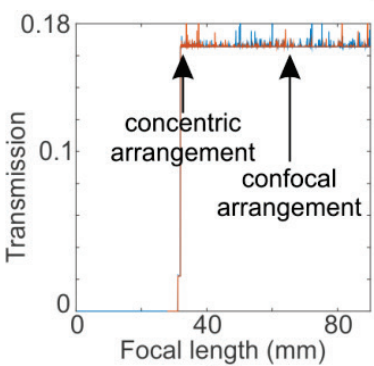

(b)

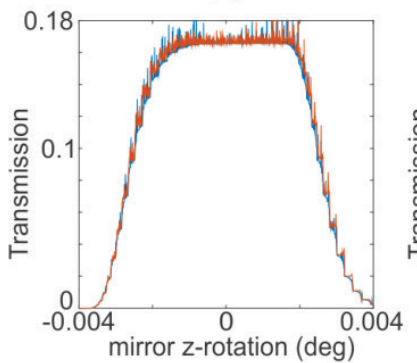

(e)

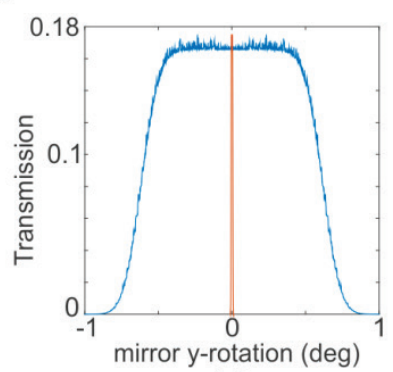

(c)

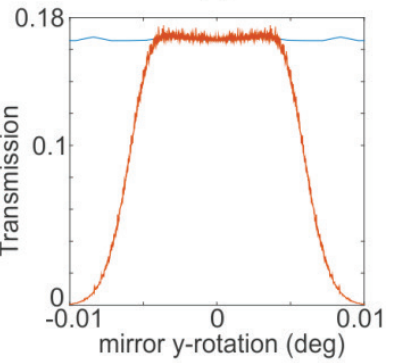

(d)
Figure 3: FRED® raytracing simulations of cylindrical mirror cells. (a) Typical setup of the simulations, consisting of a collimated light source, an off-axis parabolic (OAP) mirror for focussing, a cylindrical mirror with an absorption mask inside, and a detector collecting the light after leaving the cell. (b) Calculated transmission of parabolic (blue) and toroidal (orange) surface as a function of focal length in sagittal direction. (c) Calculated transmission of a confocal parabolic (blue) and a concentric toroidal (orange) cylindric cell under rotation of the OAP around the y-axis, with (d) a zoom-in on the same data. (e) Transmission under rotation of the OAP around the $z$-axis.

The alignment requirements in the tangential direction were studied in a similar way by rotating the coupling mirror around the zaxis, which leads to a change of the incidence angle $\Theta$ [Fig. 3(e)]. We find a tolerance of this angle of approximately $+/-0.002 \mathrm{deg}$, which is independent of the mirror arrangement or surface shape. Although this tolerance may seem tight, it can readily be achieved with standard opto-mechanical components.

Because of the higher alignment stability and the increased manufacturing tolerance of the confocal mirror arrangement, we decided to manufacture a circular mirror cell with a confocal mirror arrangement. Furthermore, we chose a parabolic mirror for the following reasons: (1) in a confocal arrangement, a beam with the focal point at the center of the cavity alternates between a collimated beam and a focalized beam; for focalizing collimated beams, a parabolic shape is ideal because it corrects for spherical aberration; (2) a parabolic shape has aberration- free focusing properties, even under non-centric 
illumination. This allows for additional beam patterns, which we refer to as off-axis star patterns.

The manufacturing parameters were chosen as follows: a diameter $D=144 \mathrm{~mm}$ and the quadratic coefficient $a=0.00380 \mathrm{~mm}^{-1}$. The manufacturing process consisted of three steps: (1) production of the steel (invar) body of the cell with the inner surface diamond turned; (2) electroless NiP coating $(150 \mu \mathrm{m})$ with subsequent diamond turning to the exact surface shape (at this stage, the surface inaccuracy was determined to be $<1 \mu \mathrm{m}$ and the surface roughness $<4 \mathrm{~nm}$ RMS); (3) $500 \mathrm{~nm}$ galvanic gold coating as a reflection layer. Unfortunately, the surface roughness after the galvanic gold coating could not be measured with our means.

In Fig. 4(a) we show a simulation of the 89-spiked star-pattern with density $q=36$ produced by irradiating such a parabolic cylindrical mirror with an incidence angle of $\Theta=17.191 \mathrm{deg}$. The same cylindrical surface can be irradiated with a beam that is offset from the central plane of the cylinder. The resulting off-axis star-pattern is shown in Fig. 4(b). The spots, where the laser beam is reflected from the surface, are now distributed on two planes, whereby the distance of adjacent reflections is increased. Such a beam pattern cannot be stably produced with a spherical surface, since spherical aberration is huge for non-centric illumination.

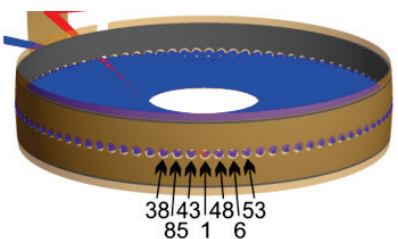

(a)

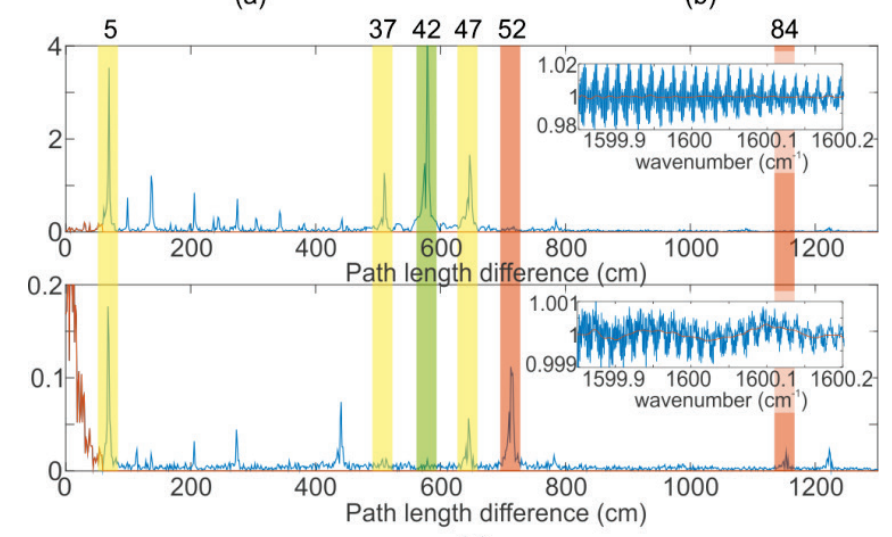

(c)

Figure 4: On- and off-axis beam patterns. FRED® simulations of the light propagation in a parabolic cell under (a) central irradiation and (b) off-axis irradiation. The numbers inidicate for a few reflection spots the number of passes through the cell the light travels to reach the respective spot. (c) Mid-infrared spectra (as insets) and their Fourier transform measured through a cylindrical cell under central irradiation (upper panel) and under off-axis irradiation (lower panel). Colored bars indicate fringe components which are fully (green), partially (yellow), or not at all (red) suppressed in the off-axis configuration. The numbers represent the number of passes through a $144 \mathrm{~mm}$ diameter cell that is necessary to reach the respective path length difference.

Optical characterization of the multipass cell is performed using a quantum cascade laser (QCL) emitting light of $6.25 \mu \mathrm{m}$ wavelength packaged in an HHL housing with integrated collimation lens. The collimated laser beam is focalized with an $f=150 \mathrm{~mm} \mathrm{CaF}_{2}$ lens with the focus positioned half way through the first pass of the star pattern. Inside the cylindrical cell is an absorption mask, i.e. a second cylinder made from polyoxymethylene (POM) with 89 holes at the position, where reflections from the mirror are expected. The light leaving the cell is collected on an $f=50 \mathrm{~mm}$ concave gold mirror and is measured using a commercial MCT detector (Vigo Systems, PL).

The transmission signal measured under in-plane irradiation resulting in a $2 \mathrm{D}$ beam pattern is shown in the inset of the upper panel of Fig. 4(c). The spectrum is dominated by strong interference fringes with amplitude of approximately $+/-2 \%$ of the mean signal. The periodicity of the observed fringes is an accurate measure of the pathlength difference of the interfering beams, which can be investigated in more detail using the Fourier transform of the measured spectrum [upper panel of Fig. 4(c)]. We observe strong peaks at clearly defined path-length differences. The strongest fringe component corresponds to an optical path difference of $575 \mathrm{~cm}$, i.e., exactly to 42 passes through the multipass cell. In Fig. 4(a) the arrows indicate for a few reflection spots how many passes the light has traveled before reaching them. For example, the position of the firstreflection is neighbored to its left by the 43rd reflection, corresponding to a path length difference of 42 passes. In fact, for more than half of the reflections, the left neighbor has a path length difference of 42 passes. This explains the large amplitude of the corresponding fringe structure at $575 \mathrm{~cm}$ and indicates some optical "spillover," i.e., the light leaks into the neighboring hole. Similarly, we can attribute the other fringe components to various path length differences, e.g., the fringe component at $644 \mathrm{~cm}$ (47 passes) corresponds for nearly half of the reflections to the path length difference to the right neighbor. We can even see contributions from the second and third nearest neighbors, as, for example, the fringe components at $68.5 \mathrm{~cm}$ (5 passes) or at $507 \mathrm{~cm}$ (37 passes). The origin of the observed "spillover" is not fully understood. Plausible causes are either stray light or imperfect imaging of the beam.

An advantage of the rather low density $(q=36)$ star-pattern is that neighboring reflections have relatively large path length differences. These lead to a small spectral width of the interference fringes, e.g., $0.015 \mathrm{~cm}^{-1}$ for the fringe component corresponding to five passes. Thus, we can effectively remove the spectral components below $0.017 \mathrm{~cm}^{-1}$ from the spectrum with a low-pass filter without distorting the absorption spectrum. In Fig. 4(c), the orange line represents the lowpass filtered data in both the spectrum and the Fourier transform. This approach leads to an approximately tenfold reduction of the fringes, leaving spectral variations on the order of $+/-1 \% 0$.

Further reduction of the interference fringes can be achieved by using an off-axis beam pattern. To that end, we only replace the absorption mask by a mask that has the 89 holes distributed in two layers to allow off-center reflections from the circular mirror, as is shown in Fig. 4(b). A spectrum and its Fourier transform measured in the off-axis configuration are plotted in the lower panel of Fig. 4(c). In this case, most of the fringes have amplitudes that are twentyfold lower compared with the in-plane configuration. Correspondingly, the plot scaling was adjusted by this factor for better comparison. Most pronounced is the reduction of the component corresponding to 42 passes, which is largely removed. The reason for this effective suppres- sion is the displacement of the spot locations to different planes. All reflections, where the left-hand neighbor has a path length difference of 42 passes, are now in different planes, c.f. $1 \rightarrow 43,6 \rightarrow 48,43 \rightarrow 85$. As a result, the spillover is hindered; thus, the interference is removed from the spectrum. Other fringe components, e.g., those at 5, 37, or 47 passes, are also significantly suppressed, although not as completely as the component at 42 passes. Neighbors having respective path length differences have also moved to different planes but only in half of the cases. Thereby, those fringe components are partially suppressed. Finally, there are fringe components, e.g., those with 52 or 84 passes difference, which are not influenced at all; they appear to have become stronger because of the different scales but are just not suppressed. Even in off-axis configuration, these reflections are always on the same plane.

In summary, the overall fringe amplitude in the off-axis configuration amounts to $1 \%$ and can be reduced to approximately $0.1 \%$ by a lowpass filter as previously mentioned.

Using this optical configuration and a setup as described in [19], the optical performance of the parabolic multipass cell is demonstrated with trace gas measurements in ambient air samples. Fig. 5(a) shows the absorption spectrum of ambient air containing $40 \mathrm{ppb} \mathrm{NO}_{2}$. The blue trace represents the measured transmission signal through the 
multipass cell filled with air at a pressure of $126 \mathrm{hPa}$. The Voigt fit (orange trace) to the most prominent $\mathrm{NO}_{2}$ and water absorption lines is obtained using parameters from the HITRAN database [26]. The fit residual [upper panel of Fig. 5(a)] reflects the $0.1 \% 0$ optical fringe level in the measured transmission. The Allan deviation plotassociated to the $\mathrm{NO}_{2}$ concentration measurement [27] indicates a measurement precision below $1 \mathrm{ppb}$ at $1 \mathrm{~s}$ averaging and below $100 \mathrm{ppt}$ at $100 \mathrm{~s}$ averaging.

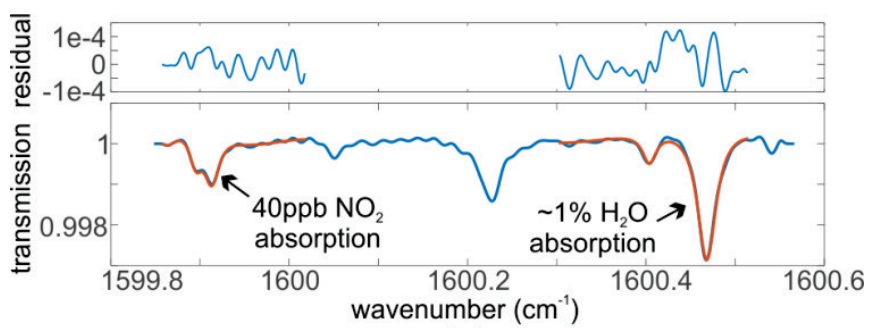

(a)

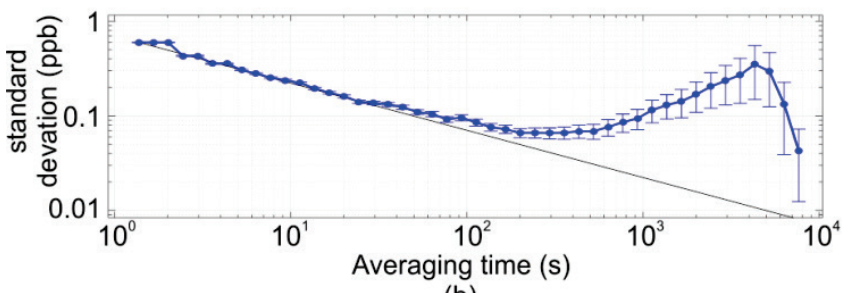

(b)

Figure 5: Trace gas measurements. (a) Low-pass filtered transmission spectrum measured in an 89-pass off-axis star-pattern geometry in air containing $40 \mathrm{ppb}$ $\mathrm{NO}_{2}$ and $1 \%$ water (blue line) and the corresponding Voigt fit using the paramters from the HITRAN database. The upper panel shows the residual transmission. (b) Allan deviation plot of the $\mathrm{NO}_{2}$ concentration measurements.

In summary, we described and validated a circular multipass reflection cell. We discussed the main design parameters, which are the optical beam pattern, the surface shape of the circular mirror, and the respective focal length of the mirrors. The simulation results illustrate that a confocal mirror arrangement is more stable and more tolerant toward manufacturing inaccuracies or other changes in surface shape, e.g., caused by thermal expansion. This makes the described cell particularly advantageous in applications where the temperature is not well controlled. Furthermore, we have shown that a parabolic mirror shape facilitates off-axis beam patterns, where reflections are distributed on different planes on the mirror surface.

To further assess the optical performance, we realized a circular multipass cell with a confocal, parabolic mirror arrangement. The cell was designed for 89 reflections, resulting in a total optical path of 12.24 $\mathrm{m}$. The in-plane configuration revealed strong optical noise of several percent of the mean signal caused by interference fringes. Analysis of the fringe spectra allowed identifying the distances generating the fringe patterns and gaining a better understanding of the involved factors. Using an off-axis beam pattern, the interference fringes could be reduced by more than an order of magnitude. We attribute this to the reduction of "spillover" to the neighboring reflections. By this, we were able to suppress the fringe level to roughly $0.1 \%$ of the overall signal. Finally, we show the cell's suitability for high-precision trace gas measurements with a detection limit below $0.1 \mathrm{ppb}$ for $\mathrm{NO}_{2}$ at ambient concentrations.

Funding. NanoTera Project IrSens II; Swiss National Science Foundation (SNSF); FOEN Innovation Funds.

Acknowledgment. The authors thank R. Brönnimann and A. Kunz for their support in electronics design. Ivo Strebel is acknowledged for helpful discussions.

\section{References}

1. M. Baudelet, Laser Spectroscopy for Sensing (Woodhead Publishing, 2014), pp. 522-543.

2. L. Ciaffoni, R. Peverall, and G. A. D. Ritchie, "Laser spectroscopyon volatile sulfur compounds: possibilities for breath analysis," J.Breath Res. 5, 024002 (2011).

3. C. Wang and P. Sahay, "Breath analysis using laser spectroscopic techniques: breath biomarkers, spectral fingerprints, and detection limits," Sensors 9, 8230-8262 (2009).

4. J. B. McManus, M. S. Zahniser, D. D. Nelson, Jr., J. H. Shorter, S. Herndon, and E. Wood, "Application of quantum cascade lasers to high-precision atmospheric trace gas measurements," Opt. Eng. 49, 111-124 (2010).

5. F. Adler, M. J. Thorpe, K. Cossel, and J. Ye, "Cavity-enhanced direct frequency comb spectroscopy: technology and applications," Annu. Rev. Anal. Chem. 3, 175-205 (2010).

6. J.U.White, "Long optical paths oflarge aperture," J. Opt. Soc. Am. 32, 285-288 (1942)

7. D. Herriott, H. Kogelnik, and R. Kompfner, "Off-axis paths in spherical mirro interferometers," Appl. Opt. 3, 523-526 (1964).

8. D. R. Herriott and H. J. Schulte, "Folded optical delay lines," Appl. Opt. 4, 883-889 (1965).

9. J. B. McManus, P. L. Kebabian, and M. S. Zahniser, "Astigmatic mirror multipass absorption cells for long-path-length spectroscopy," Appl. Opt. 34, 3336-3348 (1995).

10. S. So and D. Thomazy, "Split spherical mirror configuration for optical multipass cell," U.S. patent 2012/0092782 A1(April 19, 2012).

11. M. Thoma, R. Kaschow, and F. Hindelang, "A multiple-reflection cell suited for absorption measurements in shock tubes," Shock Waves 4, 51-53 (1994).

12. Y. Kato, S. Sato, T. Nakamura, and M. Endo, "Performance character- istics of a compact cylindrical multi-pass cell for laser absorption based trace gas sensor," in Conference on Lasers and Electro- Optics-Pacific Rim, Seoul, South Korea (2007), p. 1.

13. A. Manninen, B. Tuzson, H. Looser, Y. Bonnetti, and L. Emmenegger, "Versatile multipass cell for laser spectroscopic trace gas analysis," Appl. Phys. B 109, 461466 (2012).

14. M. Tonomura, H. Miyazawa, T. Nakamura, M. Endo, S. Yamaguchi, K. Nanri, and T. Fujioka, "An experimental study on a cylindrical multi- pass cell," in Pacific Rim Conference on Lasers and Electro-Optics (IEEE, 2007), p. 1425

15. D. Knox, A. King, E. McNaghten, S. Brooks, P. Martin, and S. Pimblott, "Novel utilisation of a circular multireflection cell applied to materials ageing experiments," Appl. Phys. B 119, 55-64 (2015)

16. S. Chernin, "New generation of multipass systems in high re-solution spectroscopy," Spectrochim. Acta Part A 52, 1009-1022 (1996).

17. J. Riedel, S. Yan, H. Kawamata, and K. Liu, "A simple yet effective multipass reflector for vibrational excitation in molecular beams," Rev. Sci. Instrum. 79, 033105 (2008).

18. J. Ofner, H.-U. Krüger, and C. Zetzsch, "Circular multireflection cell for optical spectroscopy," Appl. Opt. 49, 5001-5004 (2010).

19. B. Tuzson, M. Mangold, H. Losser, A. Manninen, and L. Emmenegger, "Compact multipass optical cell for laser spectros- copy," Opt. Lett. 38, 257-259 (2013).

20. B. Bernacki, "Multipass optical device and process for gas and analyte determination," U.S. patent 7,876,443 B2 (January 25, 2011).

21. P. Jouy, M. Mangold, B. Tuzson, L. Emmenegger, Y.-C. Chang, L. Hvozdara, H. P. Herzig, P. Wägli, A. Homsy, N. F. d. Rooij, A. Wirthmüller, D. Hofstetter, H. Looser, and J. Faist, "Mid-infrared spectroscopy for gases and liquids based on quantum cascade tech- nologies," Analyst (Amsterdam) 139, 2039-2046, (2013).

22. H. Coxeter, Introduction to Geometry (Wiley, 1989).

23. W. Demtröder, Laser Spectroscopy (Springer, 2008).

24. W. Haynes, CRC Handbook of Chemistry and Physics (Taylor \&Francis, 2012).

25. M. Mangold, B. Tuzson, and L. Emmenegger, "Method for reducing fringes in lase spectroscopy measurements using an absorption mask in combination with multipass optical cells," Switzerland patent 01884/12 (October 8, 2012)

26. L. S. Rothman, I. E. Gordon, A. Barbe, D. Chris Benner, P. F. Bernath, Birk, V. Boudon, L. R. Brown, A Campargue, J.-P. Champion, K Chance, L. H. Coudert V Dana, V. M. Devi, S. Fally, J.-M. Flaud, R. R. Gamache, A. Goldman, D. Jacquemart, I. Kleiner N. Lacome, W. J. Lafferty, J.-Y. Mandin, S. T. Massie, S. N. Mikhailenko, C. E. Miller, Moazzen-Ahmadi, O. V. Naumenko, A. V. Nikitin, J. Orphal, V. I. Perevalov A. Perrin, A. Predoi-Cross, C. P. Rinsland, M. Rotger, M. Šimečková, M. A. H. Smith, K. Sung, S. A. Tashkun, J. Tennyson, R. A. Toth, A. C. Vandaele, and J. Vander Auwera, "The HITRAN 2008 molecular spectroscopic database," J. Quant. Spectrosc. Radiat. Transfer 110, 533-572 (2009).

27. P. Werle, R. Mücke, and F. Slemr, "The limits of signal averaging in atmospheric trace-gas monitoring by tunable diode-laser absorption spectroscopy (TDLAS)," Appl. Phys. B 57, 131-139 (1993). 